\title{
Improved Release and Metabolism of Flavonoids by Steered Fermentation Processes: A Review
}

\author{
Nguyen Thai Huynh ${ }^{1,2,3,4}$, John Van Camp ${ }^{2}$, Guy Smagghe ${ }^{3}$ and Katleen Raes ${ }^{1, *}$ \\ 1 Department of Industrial Biological Sciences, Faculty of Bioscience Engineering, \\ Ghent University-Campus Kortrijk, Graaf Karel de Goedelaan 5, 8500 Kortrijk, Belgium; \\ E-Mail: thainguyen.huynh@ugent.be \\ 2 Department of Food Safety and Food Quality, Faculty of Bioscience Engineering, Ghent University, \\ Coupure Links 653, 9000 Ghent, Belgium; E-Mail: john.vancamp@ugent.be \\ 3 Department of Crop Protection, Faculty of Bioscience Engineering, Ghent University, \\ Coupure Links 653, 9000 Ghent, Belgium; E-Mail: guy.smagghe@ugent.be \\ 4 Faculty of Food Technology, Ho Chi Minh City University of Food Industry, 140 Le Trong Tan, \\ Tay Thanh Ward, Tan Phu District, 700000 Ho Chi Minh City, Vietnam
}

* Author to whom correspondence should be addressed; E-Mail: katleen.raes@ugent.be; Tel.: +32-56-241-255; Fax: +32-56-241-224.

External Editor: Antonio Segura-Carretero

Received: 9 September 2014; in revised form: 16 October 2014 / Accepted: 20 October 2014 / Published: 24 October 2014

\begin{abstract}
This paper provides an overview on steered fermentation processes to release phenolic compounds from plant-based matrices, as well as on their potential application to convert phenolic compounds into unique metabolites. The ability of fermentation to improve the yield and to change the profile of phenolic compounds is mainly due to the release of bound phenolic compounds, as a consequence of the degradation of the cell wall structure by microbial enzymes produced during fermentation. Moreover, the microbial metabolism of phenolic compounds results in a large array of new metabolites through different bioconversion pathways such as glycosylation, deglycosylation, ring cleavage, methylation, glucuronidation and sulfate conjugation, depending on the microbial strains and substrates used. A whole range of metabolites is produced, however metabolic pathways related to the formation and bioactivities, and often quantification of the metabolites are highly underinvestigated. This strategy could have potential to produce extracts with a high-added value from plant-based matrices.
\end{abstract}


Keywords: fermentation; flavonoids; bioconversion; metabolism

\section{Introduction}

Phenolic compounds are secondary metabolites produced in plants by the shikimic acid pathway. They consist of a variety of structures divided into a number of main groups including phenolic acids, flavonoids, lignans and stilbenes depending on the number of benzene rings as well as on their structure of the carbon skeleton [1]. Among these, flavonoids are the most widespread group of phenolic compounds and are further divided into different subclasses i.e., chalcones, flavonols, flavones, flavanones, flavanols, isoflavones and anthocyanins, based on various substitution patterns on rings A and $\mathrm{B}$ as well as variations in ring $\mathrm{C}$. The second most popular group of phenolic compounds are phenolic acids, which can be divided into two categories: hydroxybenzoic acids and hydroxycinnamic acids. The most common hydroxybenzoic acids are gallic acid, salicylic acid, vanillic acid, protocatechuic acid and $p$-hydroxybenzoic acid, while $p$-coumaric acid, caffeic acid, sinapic acid and ferulic acid are the best known hydroxycinnamic acids $[2,3]$.

There are several methods including physical, physicochemical and chemical techniques to extract phenolic compounds from plant and food products such as cold pressing, supercritical fluid extraction and organic solvent extraction [4], ultrasound-assisted extraction [5] and microwave-assisted extraction [6]. The disadvantage of these techniques is their low extraction yield of bound phenolic compounds, as these are mostly linked to plant cell wall material through a-OH group ( $O$-glycosides) or carbon-carbon bonds ( $C$-glycosides) [7]. As a consequence, the application of a hydrolysis step prior to conventional extraction could be used to maximize the extraction yield. Among these, enzymatic treatments and/or fermentation are preferred instead of the utilization of chemical pretreatments (acids or alkaline) as the latter results in hazards and toxicological effects due to the use of chemical products, in negative effects on the environment, and of unwanted transformations of the extracted compounds [8].

It has already been demonstrated that phenolic compounds have a wide range of bioactivities including antioxidative, anticarcinogenic, anti-inflammatory properties which are mostly related to the potential health-promoting benefits against human health risks such as hypertension, obesity, cardiovascular diseases, diabetes and cancer [9-11]. Recently, more and more evidence is available indicating that the different classes and structures of phenolic compounds result in considerable variations in their bioactivities, bioaccessibility, bioavailability as well as their metabolism in the human gastrointestinal system [1]. Heim et al. [12] reported that the phenolic aglycones have a higher antioxidative activity than their glycosides. Aglycones can be effectively absorbed through the small intestine, while the glycosidic forms, due to their complex structures and large molecular weights, reach the large intestine and are metabolized by human gut microbiota into different more simplified metabolites, which can be later absorbed $[1,13,14]$. Currently, phase I/II metabolites of phenolic compounds such as deglucosides [15-17], sulfoconjugates [18,19] and glucuronides [20], can be obtained by a fermentation process. It is therefore interesting to use bacterial or fungal fermentation processes, which not only enhance the release of bound phenolic compounds from the plant cell walls, but also convert phenolic compounds into different metabolites, which can exert other bioactivities. In this paper, release of 
phenolic compounds indicates the phenolic compounds obtained in a soluble free form in the fermentation medium. This contributes to the production of extracts and food products with a high added value.

Although several reviews are available dealing with different aspects of phenolic compounds [3,7,21-23], as far as we now there is no review focusing on microbial conversion of phenolic compounds into new metabolites by a steered fermentation process, i.e., by a controlled fermentation process adding a pure microbial strain. The aim of this paper is thus to provide an overview of the profile in phenolic compounds and their metabolites obtained by microbial fermentation from plant material with the final aim to obtain (purified) extracts rich in phenolic compounds. The microbial fermentation processes which occur during digestion fall outside the scope of this paper. The effect of microorganisms (bacteria, yeast and fungi) used in fermentation processes to release phenolic compounds from plant matrices in the fermentation media as well as the possible metabolic pathways of flavonoids (glycosylation, deglycosylation, ring cleavage, methylation, glucuronidation, and sulfate conjugation) to new conversion products are summarized and discussed.

\section{The Release of Phenolic Compounds from Plant Matrices by Fermentation Processes}

Phenolic compounds present in plants can be classified into free phenolic compounds found in the vacuoles of plant cells, and bound phenolic compounds linked to cell wall structure components (cellulose, hemicellulose, lignin, pectin and protein) through several covalent bounds [24-26]. Free phenolic compounds can be effectively extracted by conventional techniques, while several hydrolysis processes have been used to enhance the release of bound phenolics. Fermentation has been considered as one of the best processes to obtain extracts with a high quality and a high activity, using economically and environmental friendly techniques [3].

Several studies reported that fermentation influences the phenolic profile of extracts obtained from various plant sources or during the fermentation of plant sources. Table 1 summarizes the published papers between 2000 and 2014, available on the Web of Science, including the phenolic profile changes, only measured by chromatographic techniques, and obtained by a steered fermentation process (no spontaneous or natural fermentations). Vattem et al. [27,28] have found that solid-state fermentation of cranberry pomace using a food-grade fungus Lentinus edodes resulted in a maximum of a $49 \%$ increase in ellagic acid content after five days of incubation. Another study demonstrated that the phenolic acid profile in an ethanolic extract from oat fermented by three different filamentous fungi (Aspergillus oryzae var. effuses, Aspergillus oryzae and Aspergillus niger) at $25^{\circ} \mathrm{C}$ for three days was remarkably improved in comparison with non-fermented oat [29]. Indeed, the study showed that fermentation of oat using Aspergillus oryzae var. effuses or Aspergillus oryzae increased the content of caffeic acid and ferulic acid in oat (Avena sativa L.) up to about 2.7- to three-fold and 5.5- to nine-fold, respectively, when compared to native oat. Fermentation with Aspergillus oryzae var. effuses also resulted in a more than $100 \%$ increase of chlorogenic and $p$-coumaric acids. In a recent study, Schmidt et al. [30] investigated the effect of solid-state fermentation by Rhizopus oryzae on the profile of phenolic acids derived from rice bran. The content of chlorogenic acid, $p$-hydroxybenzoic acid and vanillin significantly increased during fermentation. According to these authors, an incubation for $120 \mathrm{~h}$ at $30{ }^{\circ} \mathrm{C}$ with Rhizopus oryzae led to the most substantial increase in gallic acid and ferulic acid content, ranging from 3 and $33 \mathrm{mg} / \mathrm{g}$ dried weight in native bran to 155 and $765 \mathrm{mg} / \mathrm{g}$ dried weight in fermented bran, respectively. 
Table 1. The effect of microbial fermentation on the increase in phenolic compounds from various plant-based foods.

\begin{tabular}{|c|c|c|c|}
\hline Microorganism & Source & Phenolic profile & Reference \\
\hline \multicolumn{4}{|l|}{ Bacteria } \\
\hline Bacillus pumilus & Soybean & Gallic acid, catechin, epicatechin & {$[31]$} \\
\hline Bacillus subtilis & Soybean & Chlorogenic acid, naringin & {$[32]$} \\
\hline Bacillus subtilis & Cheonggukjang (soybean paste) & Daidzein, genistein & {$[33,34]$} \\
\hline Lactobacillus acidophilus & Apple juice & Gallic acid & {$[35]$} \\
\hline $\begin{array}{l}\text { Lactobacillus johnsonii, Lactobacillus reuteri, } \\
\text { Lactobacillus acidophilus }\end{array}$ & Whole grain barley, oat groat & Sinapic acid, caffeic acid, $p$-coumaric acid, ferulic acid & {$[36]$} \\
\hline Lactobacillus plantarum & Cowpeas & Quercetin & {$[37]$} \\
\hline $\begin{array}{l}\text { Lactobacillus plantarum, } \\
\text { Lactobacillus delbrueckii supsp. lactis }\end{array}$ & Soybean & Daidzein, genistein & {$[38]$} \\
\hline \multicolumn{4}{|l|}{ Yeast } \\
\hline Saccharomyces cerevisiae & Wheat bran & Syringic acid, $p$-coumaric acid, ferulic acid & [39] \\
\hline \multicolumn{4}{|l|}{ Fungi } \\
\hline Aspergillus oryzae, Monascus purpureus & Soybean & Daidzein, genistein & [40-42] \\
\hline $\begin{array}{l}\text { Aspergillus oryzae var. effuses, } \\
\text { Aspergillus oryzae, Aspergillus niger }\end{array}$ & Oat (Avena sativa $\mathrm{L})$. & $\begin{array}{l}\text { Chlorogenic acid, ferulic acid, } p \text {-coumaric acid, } \\
\text { caffeic acid }\end{array}$ & [29] \\
\hline Aspergillus oryzae & Green tea & $\begin{array}{l}\text { Gallic acid, gallocatechin, epigallocatechin, epicatechin, } \\
\text { 3-p-coumaroylquinic acid, kaempferol-rutinoside }\end{array}$ & {$[43]$} \\
\hline Lentinus edodes & Cranberry pomace (Vaccinium acrocarpon) & Ellagic acid & {$[27,28]$} \\
\hline Rhizopus oryzae & Rice bran & $\begin{array}{l}\text { Gallic acid, ferulic acid, } p \text {-hydroxybenzoic acid, } \\
\text { caffeic acid, chlorogenic acid, vanillin }\end{array}$ & {$[30]$} \\
\hline Rhizopus oligosporus, Rhizopus oryzae & Black soybean & Daidzein, genistein & {$[44]$} \\
\hline
\end{tabular}


In addition to phenolic acids, the enhancement of the flavonoid content has also been observed in recent studies. Soybeans incubated with Aspergillus oryzae at $30{ }^{\circ} \mathrm{C}$ for $48 \mathrm{~h}$ resulted in a 23 -fold increase in genistein aglycones when compared to the content found in unfermented soybean flour [40]. The amount of these aglycones was also found to be higher in solid-state fermentations of soybean with Rhizopus sp. [44] and Monascus purpureus [41] compared to unfermented soybeans.

Similar to filamentous fungus, different food-graded lactic acid bacteria (LAB) and Bacillus spp. have been evaluated for their potential to release phenolic acids as well as flavonoids from plant sources such as soybean [31,32], apple [35] and cereals [36]. The fermentation with Lactobacillus johnsonii, Lactobacillus reuteri and Lactobacillus acidophilus showed a 20-fold increase in the content of total free phenolic acids in both barley and oat flour, compared to the unfermented sample, with the largest increase observed for free ferulic acid up to $39-56 \mu \mathrm{g} / \mathrm{g}$ dried weight depending on the strains used, while the amount of this compound found in unfermented samples was around $1 \mu \mathrm{g} / \mathrm{g}$ dried weight [36]. This study also exhibited that fermentation with Lactobacillus johnsonii had a much higher effect on the release of free phenolic acids than the other strains. A similar effect on the release of bound phenolic compounds was observed. Fermentation of grain barley with three LAB strains resulted in a significant increase of ferulic acid and $p$-coumaric acid which contributed to an increase in total content of bound phenolic acids by around $23 \%$, compared to native grain barley. Also, enhancing the release of phenolic acids and flavanols was reported in a recent study [31], showing 2.8-fold, 7.6-fold and 4.5-fold increases in gallic acid, catechin and epicatechin, respectively after $60 \mathrm{~h}$ of fermentation with Bacillus pumilus. Soybean seeds fermented with Bacillus subtilis for three days yielded an increase in chlorogenic acid and naringin [32].

Not only fungi, LAB strains and Bacillus spp. have been used, but also yeast were screened for their improvement of the free phenolic profile. Moore et al. [39] reported that solid-state fermentation of wheat bran with Saccharomyces cerevisiae yielded a maximum increase of $48 \%, 51 \%$ and $333 \%$ in the content of soluble free $p$-coumaric, ferulic and syringic acid, respectively, compared to unfermented samples, while soluble vanillic acid decreased probably due to its conversion into other metabolites.

However, a fermentation process does not exclusively increase all phenolic compounds. Also, a decrease in some components, e.g., deglycosylated, is observed, as they are metabolized into other forms having a lower toxicity towards microbial activity. Also type of microorganism, conditions of the fermentation process and fermentation time play a role herein $[42,43,45]$.

The change in the profile of phenolic compounds by the fermentation process is due to the action of cellulolytic, ligninolytic and pectinolytic enzymes, mainly produced during the growth of the microorganisms, as described in Figure 1. An overview of possible enzymes involved in the release of phenolic compounds, by breaking down the cell wall matrix and produced by the fermentation microorganisms is given in Table 2. These enzymes are known to be capable of completely breaking down the chemical components of plant cell walls, resulting in the hydrolysis of the ester bonds, which link phenolic compounds to the cell wall matrix, and in the oxidative degradation of lignin. As a consequence, the free phenolic compounds as well as bound forms are released more efficiently from the plant matrix. Among these enzymes, $\beta$-glucosidase has been widely reported as an enzyme responsible for catalyzing the hydrolysis of glycosidic linkages in alkyl and aryl- $\beta$-D-glucosides to release phenolic aglycone moieties. Vattem et al. [27] demonstrated that the increased release of the aglycone form of ellagic acid from cranberry pomace could be attributed to crude $\beta$-glucosidase 
produced, during solid-state fermentation by the food-grade fungi Lentinus edodes. Similarly, previous studies pointed out that esterases produced by filamentous fungi through solid-state fermentation of cereal sources, such as oat [29] and rice bran [30], caused an increase in the content of phenolic acids, such as ferulic acid, caffeic acid and $p$-coumaric acid.

The changes in phenolic profile were observed as a result of microbial fermentation of plant-based matrices. This confirms that the fermentation of plant substrates, both edible parts as well as agricultural by-products and food waste, with different microorganisms, including filamentous fungi, lactic acid bacteria, yeast, could be considered as a potential process to increase the release of phenolic compounds contributing to the production of extracts and food products with an added value.

Figure 1. A schematic diagram of the release and bioconversion of phenolic compounds.

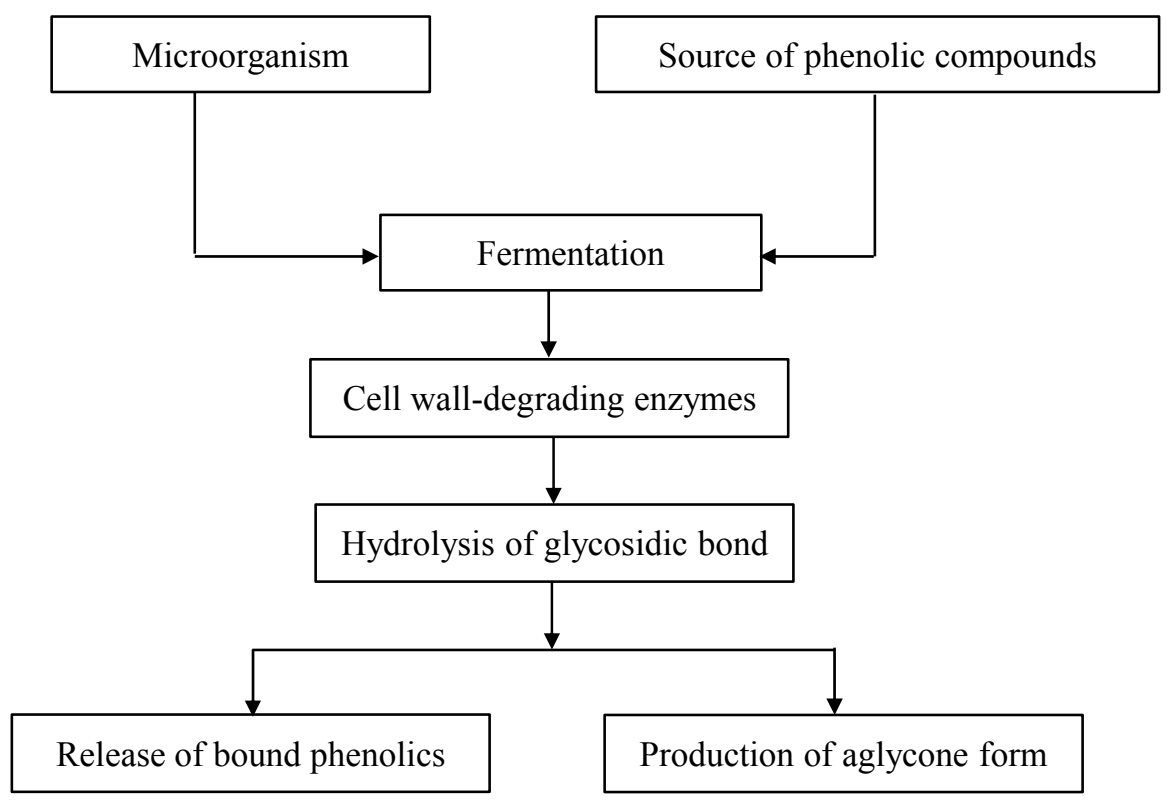

\section{Microbial Metabolism of Flavonoids during Fermentation}

Various metabolic pathways of phenolic compounds by microbial fermentation are summarized in Figure 2. The studies dealing with the bioconversion of flavonoids into their metabolites by a controlled microbial fermentation are summarized in Table 3. However, it should be mentioned that not all of the studied microorganisms are food-graded ones. Besides, only wild-type microorganisms are included, possessing the characteristics to modify phenolic compounds. Although some recent studies are investigating genetically modified organisms to obtain higher conversion yields by expressing certain enzymes, these studies are not included in this paper. A more detailed discussion of the different processes is given below. 
Table 2. Enzymes system produced by different microorganism strains to degrade the cell wall matrix.

\begin{tabular}{|c|c|c|c|}
\hline Microorganisms & Species & Enzymes & References \\
\hline \multirow{6}{*}{ Bacteria } & Lactobacillus lactis & Esterase, decarboxylase & [46] \\
\hline & Lactobacillus plantarum & $\beta$-Glucosidase, decarboxylase & [46] \\
\hline & Lactobacillus rhamnosus & Cellulase, esterase, $\beta$-glucosidase & {$[46,47]$} \\
\hline & Bacillus cereus & Cellulase, tannase & {$[46]$} \\
\hline & Bacillus subtilis & Cellulase, $\beta$-glucanase & {$[46]$} \\
\hline & Bacillus thuringiensis & Cellulase, tannase & [46] \\
\hline \multirow{9}{*}{ Fungi } & Aspergillus awamori nakazawa & Xylanase, $\alpha$-L-arabinofuranosidase, feruloyl esterase & {$[48,49]$} \\
\hline & Aspergillus niger & Cellulase, esterase, $\beta$-glucosidase, xylanase & {$[46,48-50]$} \\
\hline & Aspergillus oryzae & Cellulase, $\beta$-glucosidase, xylanase, pectinase & [51] \\
\hline & Lentinus edodes & Cellulase, $\beta$-glucosidase, xylanase, manganese peroxidase, laccase & {$[52,53]$} \\
\hline & Penicillium brasilianum & Feruoylesterase & [54] \\
\hline & Pleurotus ostreatus & Laccase, $\alpha$-/ $\beta$-glucosidase & {$[55,56]$} \\
\hline & Rhizopus oligosporus & $\beta$-glucosidase, $\beta$-glucuronidase, xylanase & [57-59] \\
\hline & Phanerochaete chrysosporium & $\beta$-Glucosidase, lignin peroxidases, manganese peroxidase, laccase & {$[60]$} \\
\hline & Rhizopus oryzae & $\beta$-glucosidase, tannase, pectinase & [24] \\
\hline \multirow{4}{*}{ Yeast } & Crytococcus flavus & $\beta$-glucosidase, $\beta$-glucanase, esterase, xylanase & [46] \\
\hline & Rhodotorula glutimis & $\beta$-Glucosidase & [46] \\
\hline & Sacharomyces cerevisiae & $\beta$-Glucosidase, feruoylesterase & {$[46,61]$} \\
\hline & Wickerhamomyces anomalus & $\beta$-Glucosidase, esterase & {$[62]$} \\
\hline
\end{tabular}


Table 3. Microbial metabolism of flavonoids through fermentation process.

\begin{tabular}{|c|c|c|c|}
\hline Substrate & Production & Microorganism & Reference \\
\hline \multicolumn{4}{|l|}{ Glycosylation } \\
\hline Quercetin & Isoquercetin (quercetin-3-glucoside) & Bacillus cereus & [63] \\
\hline Catechin & $\begin{array}{l}\text { Catechin } 7-\alpha \text {-D-glucopyranoside } \\
\text { Catechin } 5 \text { - } \alpha \text {-D-glucopyranoside }\end{array}$ & Bacillus stearothermophilus & [64] \\
\hline Luteolin & $\begin{array}{l}\text { Luteolin-3'-O- } \alpha \text {-D-glucopyranoside } \\
\text { Luteolin-4'- } O-\alpha \text {-D-glucopyranoside }\end{array}$ & Leuconostoc mesenteroides & {$[65]$} \\
\hline Catechin & Catechin-4'- $\beta$-D-fucopyranoside & Aspergillus niger & [64] \\
\hline Kaempferol & $\begin{array}{c}\text { Kaempferol 3- } \beta-O \text {-glucopyranoside } \\
\text { Kaempferol 4'-O- } \alpha \text {-L-rhamnopyranoside }\end{array}$ & Cunninghamella blakesleeana & [66] \\
\hline Kaempferol & Kaempferol 3- $\beta$-O-glucopyranoside & Cunninghamella echinulata & [67] \\
\hline Flavonol & Flavonol 3- $\beta$ - $O$-glucopyranoside & Cunninghamella echinulata & [67] \\
\hline Quercetin & Quercetin 3-O- $\beta$-D-glucopyranoside & Cunninghamella elegans & [68] \\
\hline Quercetin & Quercetin glycoside & Penicillium decumbens & [69] \\
\hline Kaempferol & Kaempferol glycoside & Penicillium decumbens & [69] \\
\hline Isorhamnetin & Isorhamnetin glycoside & Penicillium decumbens & [69] \\
\hline \multicolumn{4}{|l|}{ Deglycosylation } \\
\hline Daidzin & Daidzein & Bacillus pumilus & [31] \\
\hline Daidzin & Daidzein & Bacillus subtilis & [33] \\
\hline Kaempferol-3-O-glucoside & Kaempferol & Bifidobacterium pseudocatenulatum & [70] \\
\hline Naringin & Prunin & Clostridium stercorarium & [71] \\
\hline Quercetin-glucoside & Quercetin & Lactobacillus plantarum & [72] \\
\hline Ploridzin & Phloretin & Lactobacillus plantarum & [72] \\
\hline Kaempferol-3-rutinoside & Kaempferol, kaempferol-3-glucoside & Aspergillus awamori & [17] \\
\hline Rutin & Quercetin, quercetin-3-O-glucoside & Aspergillus awamori & [17] \\
\hline Rutin & Quercetin, quercetin-3-O-glucoside & Aspergillus niger & [73] \\
\hline Daidzin, glycitin, genistin & Daidzein, glycitein, genistein & Aspergillus oryzae & [15] \\
\hline Narigin & Naringenin & Curvularia lunata & [74] \\
\hline \multicolumn{4}{|l|}{ Ring cleavage } \\
\hline Quercetin & 2-Protocatechuoylphloroglucinol carboxylic acid & Aspergillus flavus & [2] \\
\hline Flavanone & 2'-Hydroxydibenzoylmethane & Aspergillus niger & [2] \\
\hline Quercetin & 2-Protocatechuoylphloroglucinol carboxylic acid & Asperillus niger & [75] \\
\hline
\end{tabular}


Table 3. Cont.

\begin{tabular}{|c|c|c|c|}
\hline Substrate & Production & Microorganism & Reference \\
\hline \multicolumn{4}{|l|}{ Ring cleavage } \\
\hline Flavanone & $\begin{array}{l}\text { 2'-Hydroxychalcone; 2',4-hydroxydihydrochalcone } \\
\text { 2,4-Dihydroxychalcone }\end{array}$ & Gibberella fujikuroi & {$[76]$} \\
\hline Flavanone & $\begin{array}{c}\text { 2',3",4"-Trihydroxydihydrochalcone } \\
\text { 2'-Hydroxydihydrochalcone }\end{array}$ & Penicillium chrysogenum & {$[2]$} \\
\hline \multicolumn{4}{|l|}{ Methylation } \\
\hline Quercetin & 3'-O-methylquercetin & Beauveria sp. & {$[77]$} \\
\hline Quercetin & Methylquercetin & Beauveria bassiana & {$[78]$} \\
\hline Rutin & Methylrutin & Cunninghamella echinulata & {$[78]$} \\
\hline Quercetin 3-O- $\beta$-D-glucopyranoside & Isorhamnetin 3-O- $\beta$-D-glucopyranoside & Cunninghamella elegans & {$[68]$} \\
\hline 7-Hydroxyflavanone & 7-Methoxyflavanone & Penicillium chermesinum & [79] \\
\hline & 3',4'-Dihydroxy-7-methoxyflavanone & & \\
\hline \multicolumn{4}{|l|}{ Glucuronidation } \\
\hline Quercetin & Quercetin glucuronide & Beauveria bassiana & {$[78]$} \\
\hline Rutin & Rutin glucuronide & Cunninghamella echinulata & {$[78]$} \\
\hline \multirow{4}{*}{ Quercetin } & Quercetin-4'- $O$ - $\beta$-D-glucuronide & Streptomyces sp. & {$[20]$} \\
\hline & Quercetin-3'- $O-\beta$-D-glucuronide & & \\
\hline & Quercetin-3-O- $\beta$-D-glucuronide & & \\
\hline & Quercetin-7-O- $\beta$-D-glucuronide & & \\
\hline \multirow{3}{*}{ Rutin } & Quercetin-4'-O- $\beta$-D-glucuronide & Streptomyces sp. & {$[20]$} \\
\hline & Quercetin-3-O- $\beta$-D-glucuronide & & \\
\hline & Quercetin-7-O- $\beta$-D-glucuronide & & \\
\hline \multirow[t]{2}{*}{ Naringenin } & Naringenin-7-O- $\beta$-D-glucuronide & Streptomyces sp. & {$[20]$} \\
\hline & Naringenin-4'-O- $\beta$-D-glucuronide & & \\
\hline \multicolumn{4}{|l|}{ Sulfate conjugation } \\
\hline Kaempferol & Kaempferol-4'-sulfate & Cunninghamella blakesleeana & {$[66]$} \\
\hline Rutin & Rutin sulfate & Cunninghamella echinulata & [78] \\
\hline Hesperitin & Hesperetin-7-sulfate & Mucor ramannianus & {$[80]$} \\
\hline 5-Hydroxyflavone & 5,4'-Dihydroxyflavone-4'-sulfate & Streptomyces fulvissimus & {$[2]$} \\
\hline
\end{tabular}


Figure 2. A schematic diagram of microbial conversion of phenolic compounds.

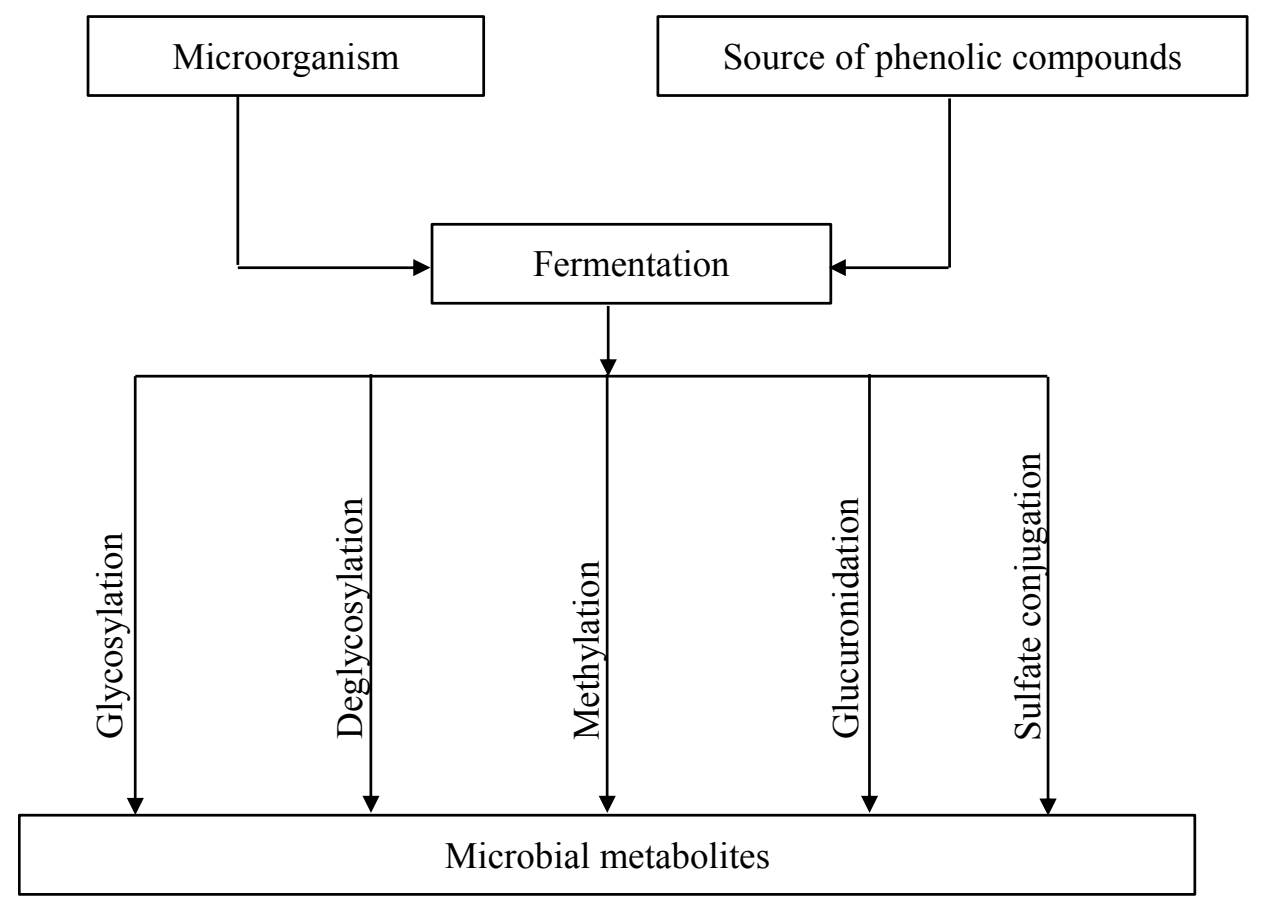

\subsection{Glycosylation and Deglycosylation of Flavonoids}

\subsubsection{Glycosylation of Flavonoids}

Glycosylation is the reaction in the biosynthesis of phenolic compounds whereby an activated glycosyl donor is attached to a phenolic aglycone through linkage to hydroxyl groups. This reaction could be performed by glycosyltransferases, resulting in a higher hydrophilic solubility of these, mainly lipophilic compounds. The glycosylation process could be applied to enhance the stabilization, detoxification and solubilization of the substrates [67]. Some microorganisms such as Bacillus cereus, Streptomyces rimosus, Cunninghamella elegans and Cunninghamella echinulata are known to be capable of glycosylating phenolic compounds [63,67,68,81]. Quercetin can be converted into isoquercetin (quercetin-3-glucoside) with a $20 \%$ bioconversion yield using a fermentation process with Bacillus cereus at $30^{\circ} \mathrm{C}$ [63]. As reported by $\mathrm{Zi}$ et al. [68], incubation of quercetin with Cunninghamella elegans ATCC9245 yielded quercetin-3-O- $\beta$-D-glucopyranoside. Similar to quercetin, kaempferol was glycosylated by the filamentous fungus Cunninghamella echinulata [67]. According to Slana et al. [82], under conditions whereby flavonoids are toxic, e.g., when they are present in high concentrations, microorganisms such as fungi could produce glycosylating enzymes (glycosyltransferase) transforming the phenolic compounds into less toxic metabolites. Not only glycolysltransferases but also other enzymes can be involved in the glycosylation of phenolic compounds, as it was shown e.g., for glucansucrase of Leuconostoc mesenteroides acting on luteolin, quercetin and myricetin [65], cellulase of Penicillium decumbrens on quercetin [69], cellulase of Aspergillus niger on catechin [64], or $\alpha$-amylase of Bacillus sp. on catechin [64]. 


\subsubsection{Deglycosylation of Flavonoids}

In contrast to glycosylation, many studies indicated that deglycosilation of phenolic compounds could be performed through microbial fermentation due to glycosyl hydrolase activities, such as $\beta$-glucosidase [28,52], naringinase [74], $\alpha$-rhamnosidase and hesperidinase [17]. Park et al. [83] reported that glucose moieties attached to flavonoids at the $\mathrm{C} 3$ and $\mathrm{C} 7$ positions can be a substrate for $\beta$-glucosidase [EC 3.2.1.21]. This enzyme is well known for its deglycosylation capability by hydrolyzing the $\beta-1,4$ glycosidic bonds in aryl and alkyl $\beta$-D-glucosides as well as glycosides containing disaccharides and oligosaccharides [52,84]. Recently, several studies for increasing the concentration of isoflavone aglycones in soy products have been performed [15,31,70]. Cho et al. [31,33] reported that fermented soybeans with Bacillus pumilus HY1 or Bacillus subtilis CS90 for 48 h incubation resulted in the highest concentration of isoflavone aglycones (daidzein) and thus in a decrease in isoflavone glucosides. This finding was also reported by Lee et al. [15], who found that soybeans fermentation by Aspergillus oryzae KACC 40247 seemed to cause a significant increase in the amount of isoflavone aglycones including daidzein, glycitein and genistein. In general, the biotransformation of glycosidic flavonoids occurring in soybeans into their corresponding aglycones during fermentation was attributed to microbial $\beta$-glucosidase activity $[15,31,33]$. This enzyme could be considered as a possible reason for the deglycosylation of kaempferol-3-O-glucoside into kaempferol through fermentation with Bifidobacterium pseudocatenulatum B7003 [70] or Aspergillus awamori [17], quercetin-3-glucoside into quercetin by Aspergillus awamori [17].

Another enzyme also produced by fungal fermentation is $\alpha$-L-rhamnosidase [EC 3.2.1.40], which cleaves terminal $\alpha$-L-rhamnose present in many natural glycosides such as naringin, rutin, quercitrin, hesperidin, diosgene, terpenyl glycosides [85]. For example, fermentation of rutin with food-grade Aspergillus niger for $4 \mathrm{~h}$ [73] or Aspergillus awamori for 4 days [17] resulted in transformation of rutin into (isoquercetin) quercetin-3-glucoside which could be attributed to $\alpha$-L-rhamnosidase activity well known for its capability of removing one rhamnose moiety. Also some $\alpha$-rhamnosidase activity was measured in different lactic acid bacteria, with the most positive strains belonging to Lactobacillus plantarum and able to release rhamnose units from hesperidin and rutin rhamnose metabolites [86].

\subsection{Ring Cleavage of Flavonoids}

Many flavonoids undergo a ring-opening reaction in which their C-ring is split and chalcones along with hydroxylations at different C-positions are produced. As shown by Udupa et al. [76], a number of hydroxylated chalcone metabolites (2'-hydroxychalcone; 2',4-dihydroxydihydrochalcone; 2',4-dihydroxychalcone) are produced when flavanone was incubated with the fungal strain, Gibberella fujikuroi. The ring fission of the heterocylic C-ring of flavanone is known to occur among several fungal species such as Aspergillus, Penicillium, Rhizopus, Monacus [2]. Incubation of unsubstituted flavanone with Aspergillus niger x172 yielded the chalcone products 2'-hydroxydibenzoylmethane and 2',3",4"-trihydroxydihydrochalcone [2]. Another strain, Penicillium chrysogenum cleaves the C-ring of flavanone into 2'-hydroxydihydrochalcone [2].

Similarly, biotransformation of quercetin to 2-protocatechuoylphloroglucinol carboxylic acid was observed by Das et al. [2], who indicated that the C-ring of quercetin was oxidized and 
cleaved by the enzyme quercetinase produced by Aspergillus flavus. The cleavage of quercetin to 2-protocatechuoylphloroglucinol carboxylic acid was also performed by flavonol 2,4-dioxygenase, an enzyme produce by Aspergillus niger DSM 821 [75].

\subsection{Methylation of Flavonoids}

$O$-Methylated flavonoids, known as xenobiotic transformation metabolites, is a common hepatic metabolite obtained by phase II reaction occurring in mammalians by $O$-methyl transferases [1]. However, some fungal species have been evaluated for their capability of methyl conjugation [68,77-79]. According to Eula Maria de et al. [77], some of Beauveria strains used in their study exhibited the ability to produce $3^{\prime}-\mathrm{O}$-methylquercetin by incubation of quercetin at $29^{\circ} \mathrm{C}$ for $72 \mathrm{~h}$. Another flavonoid, rutin was also methylated into methylrutin by Cunninghamella echinulata [78]. The fermentation of 7-hydroxyflavanone with Penicillum chermesinum 113 at $25{ }^{\circ} \mathrm{C}$ for six days resulted in two methylated products: 7-methoxyflavanone and 3',4'-dihydroxy-7-methoxyflavanone [79]. $O$-Methylation was also found in the transformation pathway of quercetin into isorhamnetin 3-O- $\beta$-D-glucopyranoside as reported by Zi et al. [68] using Cunninghamella elegans ATCC 9245 at $28^{\circ} \mathrm{C}$ for $72 \mathrm{~h}$.

\subsection{Glucuronidation of Flavonoids}

The use of metabolism of bioactive compounds with microorganisms to produce specific mammalian metabolites started a decade ago. A few microbial strains are known for their ability to produce flavonoid metabolites by glucuronidation such as Beauveria bassiana [78], Cunninghamella echinulata [78] and Streptomyces sp. [20]. Araújo et al. [78] reported that fermentation of quercetin and rutin with Beauveria bassiana and Cunninghamella echinulata respectively resulted in their corresponding glucuronide. Recently, a study has demonstrated that a fermentation process of several phenolic compounds (e.g., naringenin, rutin, quercetin) using a Streptomyces sp. can produce glucuronidated products [20]. According to these authors, quercetin incubated with a culture of Streptomyces M52104 at $28{ }^{\circ} \mathrm{C}$ for $65 \mathrm{~h}$ resulted in several glucuronidated compounds including quercetin-4'- $O-\beta$-D-glucuronide, quercetin-3-O- $\beta$-D-glucuronide and quercetin-7- $O-\beta$-D-glucuronide. Similarly, both naringenin and naringenin-7-O-glucoside were also glucuronidated into naringenin-7- $O$ - $\beta$-D-glucuronide and naringenin-4'-O- $\beta$-D-glucuronide by fermentation with Streptomyces M52104 at $28^{\circ} \mathrm{C}$ for $65 \mathrm{~h}[20]$. The microbial production of glucuronidates could be attributed to the detoxification pathways in which bioactive compounds are conjugated with glucuronic acid leading to an increased solubility and a higher molecular weight [20].

\subsection{Sulfate Conjugation of Flavonoids}

Sulfate conjugation is a major pathway for the phase II metabolism of phenolic compounds in humans via the bile using arylsulphotransferase, originating from human colonic bacteria [1]. However, recent studies have shown that bioconversion of phenolic compounds into their sulfated conjugated form could also be performed by a few fungal strains including Cunninghamella echinulata [78], Cunninghamella blakesleeana [66], Streptomyces fulvissimus [2], and Mucor ramannianus [80]. Rutin incubated with Cunninghamella echinulata induced rutin sulfate [78]. 5-Hydroxyflavone was 
converted into 5,4'-dihydroxyflavone-4'-sulfate by Streptomyces fulvissimus [2]. Ibrahim et al. [66] reported that incubation of kaempferol with Cunninghamella blakesleeana (ATCC 8688A) led to the production of kaempferol-4'-sulfate. A similar result was observed by Herath et al. [80] indicating that Mucor ramannianus (ATCC 2628) was able to convert hesperitin into hesperetin-7-sulfate.

\section{Perspectives}

From the previous sections, it becomes clear that different microorganisms can produce a whole range of metabolites. To our view there will probably be many more conversion products formed in nature, than the ones we presented here, especially as we excluded natural fermentation processes in the discussion of this review. Natural fermentation processes contain an enormous diversity in microflora, often uncharacterized, but also the potential of these microorganisms towards the metabolism of secondary plant compounds are unknown. Even with controlled fermentation processes, using well-defined microorganisms, there is a lack of knowledge on the conversion potential of phenolic compounds. So, more studies in this area are needed to elucidate the microbial pathways of flavonoid conversion, identification of the metabolites, and bioactivity determinations. However research to answer these research gaps is often hampered by the lack of flavonoid standards in quantities large enough to carry out the fermentation experiments. Of course one could use well-defined plant extracts as sources of flavonoids in the fermentation processes, but it is known that additive, synergistic and antagonistic effects exist between phenolic compounds and thus would effect the conversions. A lot of studies have been done using plant extracts, however the extraction yield and profile of the phenolic compounds can differ between extraction batches due to the high variability of the phenolic compounds present in the raw plant material. Also a detailed identification and quantification of the phenolic compounds of these extracts are often lacking, although it is necessary information. Indeed analytical tools to identify and further quantify the metabolites becomes very specialized, but crucial to understanding the microbial metabolism of flavonoids. Furthermore, in depth insight in the reasons for the conversion of flavonoids by microorganisms is really needed. Some results point out that this conversion is a detoxification mechanism, but toxic levels and mechanisms of toxicity of the newly formed metabolites are unknown. This insight will allow us to go back to the microbial metabolic pathways, giving essential information needed for the development of genetically modified microorganisms overproducing specific metabolites. However, overproduction of metabolites is only relevant if these conversion products exert a (high and/or interesting) bioactivity. Several studies are done showing antioxidative and antimicrobial activity of the aglycone flavonoid form. Only few studies have dealt with their inhibitory effect on (digestive) enzymes, coloring effects, other health effects (e.g., antihypertensive, anticarcinogenic, anti-obesity), or their function as an herbicide or insecticide. However, studies evaluating the bioactivities of microbial flavonoid metabolites (e.g., sulfated compounds, hydroxylated compounds) are rare. Therefore, more studies dealing with this research gap can lead to the discovery of a huge potential of new bioactive natural compounds.

\section{Conclusions}

In summary, increased release of phenolic compounds in fermented plant-based foods is due to the action of cell wall-degrading enzymes produced through fermentation. In addition, microbial fermentation 
can induce the bioconversion of flavonoids into their metabolites by different pathways including glycosylation, deglycosylation, ring cleavage, methylation, glucuronidation and sulfate conjugation according to microbial strains and substrates. Thus, microbial fermentation could be considered as a potential technology for releasing phenolic compounds from natural resources, as well as for producing new bioactive compounds. Although some fermentation processes are applied in the production of phenolic compounds, the yield of bioconversion is variable, depending on the fermentation parameters used (e.g., microorganisms, medium, temperature, $\mathrm{pH}$ ) as well as due to differences in the plant matrix itself. Therefore, further research into optimal process is required. Also, there is still a lack of knowledge on the metabolic pathways as well as the relationship between specific metabolites and their corresponding bioactivity, bioaccessibility and bioavailability, which thus demands more research in this field.

\section{Acknowledgments}

Nguyen T. Huynh is grateful for the PhD grant financed by MOET-Vietnam. The authors thank the Special Research Fund (BOF) of Ghent University (BOF05V02512) and the Hercules foundation (AUGE028 and AUGE014) for financial support.

\section{Author Contributions}

Nguyen Thai Huynh is a PhD student under the supervision of John Van Camp, Guy Smagghe and Katleen Raes; Nguyen Thai Huynh has searched the literature to write the review, and prepared together with Katleen Raes the first draft of the manuscript; John Van Camp and Guy Smagghe reviewed and helped to revise the manuscript.

\section{Conflicts of Interest}

The authors declare no conflict of interest.

\section{References}

1. Celep, G.S.; Rastmanesh, R.; Marotta, F. Chapter 43-Microbial metabolism of polyphenols and health. In Polyphenols in Human Health and Disease; Watson, R.R., Preedy, V.R., Zibadi, S., Eds.; Academic Press: San Diego, CA, USA, 2014; pp. 577-589.

2. Das, S.; Rosazza, J.P.N. Microbial and enzymatic transformations of flavonoids. J. Nat. Prod. 2006, 69, 499-508.

3. Martins, S.; Mussatto, S.I.; Martínez-Avila, G.; Montañez-Saenz, J.; Aguilar, C.N.; Teixeira, J.A. Bioactive phenolic compounds: Production and extraction by solid-state fermentation: A review. Biotechnol. Adv. 2011, 29, 365-373.

4. Wijngaard, H.; Hossain, M.B.; Rai, D.K.; Brunton, N. Techniques to extract bioactive compounds from food by-products of plant origin. Food Res. Int. 2012, 46, 505-513.

5. Muñiz-Márquez, D.B.; Martínez-Ávila, G.C.; Wong-Paz, J.E.; Belmares-Cerda, R.; Rodríguez-Herrera, R.; Aguilar, C.N. Ultrasound-assisted extraction of phenolic compounds from Laurus nobilis L. and their antioxidant activity. Ultrason. Sonochem. 2013, 20, 1149-1154. 
6. Švarc-Gajić, J.; Stojanović, Z.; Segura Carretero, A.; Arráez Román, D.; Borrás, I.; Vasiljević, I. Development of a microwave-assisted extraction for the analysis of phenolic compounds from Rosmarinus officinalis. J. Food Eng. 2013, 119, 525-532.

7. Acosta-Estrada, B.A.; Gutiérrez-Uribe, J.A.; Serna-Saldívar, S.O. Bound phenolics in foods: A review. Food Chem. 2014, 152, 46-55.

8. Puri, M.; Sharma, D.; Barrow, C.J. Enzyme-assisted extraction of bioactives from plants. Trends Biotechnol. 2012, 30, 37-44.

9. Rodriguez-Mateos, A.; Heiss, C.; Borges, G.; Crozier, A. Berry (poly)phenols and cardiovascular health. J. Agric. Food Chem. 2014, 62, 3842-3851.

10. Nile, S.H.; Park, S.W. Edible berries: Bioactive components and their effect on human health. Nutrition 2014, 30, 134-144.

11. Ranilla, L.G.; Kwon, Y.-I.; Apostolidis, E.; Shetty, K. Phenolic compounds, antioxidant activity and in vitro inhibitory potential against key enzymes relevant for hyperglycemia and hypertension of commonly used medicinal plants, herbs and spices in Latin America. Bioresour. Technol. 2010, 101, 4676-4689.

12. Heim, K.E.; Tagliaferro, A.R.; Bobilya, D.J. Flavonoid antioxidants: Chemistry, metabolism and structure-activity relationships. J. Nutr. Biochem. 2002, 13, 572-584.

13. Chen, H.; Sang, S. Biotransformation of tea polyphenols by gut microbiota. J. Funct. Foods 2014, 7, 26-42.

14. Francisco, J.P.-C.; Malen, M.-C.; Àngels, F.; Cristina, C.; Margarida, C. The effects of cocoa on the immune system. Front. Pharmacol. 2013, doi:10.3389/fphar.2013.00071.

15. Lee, S.-H.; Seo, M.-H.; Oh, D.-K. Deglycosylation of isoflavones in isoflavone-rich soy germ flour by Aspergillus oryzae KACC 40247. J. Agric. Food Chem. 2013, 61, 12101-12110.

16. Braune, A.; Blaut, M. Intestinal bacterium Eubacterium cellulosolvens deglycosylates flavonoid $C$ - and $O$-glucosides. Appl. Environ. Microbiol. 2012, 78, 8151-8153.

17. Lin, S.; Zhu, Q.; Wen, L.; Yang, B.; Jiang, G.; Gao, H.; Chen, F.; Jiang, Y. Production of quercetin, kaempferol and their glycosidic derivatives from the aqueous-organic extracted residue of litchi pericarp with Aspergillus awamori. Food Chem. 2014, 145, 220-227.

18. Ibrahim, A.R.; Abul-Hajj, Y.J. Aromatic hydroxylation and sulfation of 5-hydroxyflavone by Streptomyces fulvissimus. Appl. Environ. Microbiol. 1989, 55, 3140-3142.

19. Mikell, J.R.; Khan, I.A. Bioconversion of 7-hydroxyflavanone: Isolation, vharacterization and bioactivity evaluation of twenty-one phase I and phase II microbial metabolites. Chem. Pharm. Bull. 2012, 60, 1139-1145.

20. Marvalin, C.; Azerad, R. Microbial glucuronidation of polyphenols. J. Mol. Catal. B: Enzym. 2011, $73,43-52$.

21. D'Archivio, M.; Filesi, C.; Varì, R.; Scazzocchio, B.; Masella, R. Bioavailability of the polyphenols: Status and controversies. Int. J. Mol. Sci. 2010, 11, 1321-1342.

22. Pereira, D.; Valentão, P.; Pereira, J.; Andrade, P. Phenolics: From chemistry to biology. Molecules 2009, 14, 2202-2211.

23. Peres-Gregorio, M.R.; Regueiro, J.; Simal-Gandara, J.; Rodriguez, A.S.; Almeida, D.P.F. Increasing the added value of onions as a source of antioxidant flavonoids: A critical review. Crit. Rev. Food Sci. 2014, 54, 1050-1062. 
24. Yadav, G.; Singh, A.; Bhattacharya, P.; Yuvraj, J.; Banerjee, R. Comparative analysis of solid-state bioprocessing and enzymatic treatment of finger millet for mobilization of bound phenolics. Bioprocess Biosyst. Eng. 2013, 36, 1563-1569.

25. Pinelo, M.; Arnous, A.; Meyer, A.S. Upgrading of grape skins: Significance of plant cell-wall structural components and extraction techniques for phenol release. Trends Food Sci. Technol. 2006, 17, 579-590.

26. Cerda, A.; Martínez, M.E.; Soto, C.; Poirrier, P.; Perez-Correa, J.R.; Vergara-Salinas, J.R.; Zúñiga, M.E. The enhancement of antioxidant compounds extracted from Thymus vulgaris using enzymes and the effect of extracting solvent. Food Chem. 2013, 139, 138-143.

27. Vattem, D.A.; Shetty, K. Ellagic acid production and phenolic antioxidant activity in cranberry pomace (Vaccinium macrocarpon) mediated by Lentinus edodes using a solid-state system. Process Biochem. 2003, 39, 367-379.

28. Vattem, D.A.; Lin, Y.T.; Labbe, R.G.; Shetty, K. Phenolic antioxidant mobilization in cranberry pomace by solid-state bioprocessing using food grade fungus Lentinus edodes and effect on antimicrobial activity against select food borne pathogens. Innov. Food Sci. Emerg. Technol. 2004, 5, 81-91.

29. Cai, S.; Wang, O.; Wu, W.; Zhu, S.; Zhou, F.; Ji, B.; Gao, F.; Zhang, D.; Liu, J.; Cheng, Q. Comparative study of the effects of solid-state fermentation with three filamentous fungi on the total phenolics content (TPC), flavonoids, and antioxidant activities of subfractions from oats (Avena sativa L.). J. Agric. Food. Chem. 2011, 60, 507-513.

30. Schmidt, C.G.; Gonçalves, L.M.; Prietto, L.; Hackbart, H.S.; Furlong, E.B. Antioxidant activity and enzyme inhibition of phenolic acids from fermented rice bran with fungus Rizhopus oryzae. Food Chem. 2014, 146, 371-377.

31. Cho, K.M.; Hong, S.Y.; Math, R.K.; Lee, J.H.; Kambiranda, D.M.; Kim, J.M.; Islam, S.M.A.; Yun, M.G.; Cho, J.J.; Lim, W.J.; et al. Biotransformation of phenolics (isoflavones, flavanols and phenolic acids) during the fermentation of cheonggukjang by Bacillus pumilus HY1. Food Chem. 2009, 114, 413-419.

32. Chung, I.-M.; Seo, S.-H.; Ahn, J.-K.; Kim, S.-H. Effect of processing, fermentation, and aging treatment to content and profile of phenolic compounds in soybean seed, soy curd and soy paste. Food Chem. 2011, 127, 960-967.

33. Cho, K.M.; Lee, J.H.; Yun, H.D.; Ahn, B.Y.; Kim, H.; Seo, W.T. Changes of phytochemical constituents (isoflavones, flavanols, and phenolic acids) during cheonggukjang soybeans fermentation using potential probiotics Bacillus subtilis CS90. J. Food Comp. Anal. 2011, 24, 402-410.

34. Shin, E.-C.; Lee, J.; Hwang, C.; Lee, B.; Kim, H.; Ko, J.; Baek, I.-Y.; Shin, J.; Nam, S.; Seo, W.; et al. Enhancement of total phenolic and isoflavone-aglycone contents and antioxidant activities during Cheonggukjang fermentation of brown soybeans by the potential probiotic Bacillus subtilis CSY191. Food Sci. Biotechnol. 2014, 23, 531-538.

35. Ankolekar, C.; Johnson, K.; Pinto, M.; Johnson, D.; Labbe, R.G.; Greene, D.; Shetty, K. Fermentation of whole apple juice using lactobacillus acidophilus for potential dietary management of hyperglycemia, hypertension, and modulation of beneficial bacterial responses. J. Food Biochem. 2012, 36, 718-738. 
36. Hole, A.S.; Rud, I.; Grimmer, S.; Sigl, S.; Narvhus, J.; Sahlstrøm, S. Improved bioavailability of dietary phenolic acids in whole grain barley and oat groat following fermentation with probiotic Lactobacillus acidophilus, Lactobacillus johnsonii, and Lactobacillus reuteri. J. Agric. Food Chem. 2012, 60, 6369-6375.

37. Dueñas, M.; Fernández, D.; Hernández, T.; Estrella, I.; Muñoz, R. Bioactive phenolic compounds of cowpeas (Vigna sinensis L): Modifications by fermentation with natural microflora and with Lactobacillus plantarum ATCC 14917. J. Sci. Food Agric. 2005, 85, 297-304.

38. Pyo, Y.-H.; Lee, T.-C.; Lee, Y.-C. Effect of lactic acid fermentation on enrichment of antioxidant properties and bioactive isoflavones in soybean. J. Food Sci. 2005, 70, S215-S220.

39. Moore, J.; Cheng, Z.; Hao, J.; Guo, G.; Liu, J.-G.; Lin, C.; Yu, L. Effects of solid-state yeast treatment on the antioxidant properties and protein and fiber compositions of common hard wheat bran. J. Agric. Food Chem. 2007, 55, 10173-10182.

40. Da Silva, L.H.; Celeghini, R.M.S.; Chang, Y K. Effect of the fermentation of whole soybean flour on the conversion of isoflavones from glycosides to aglycones. Food Chem. 2011, 128, 640-644.

41. Handa, C.L.; Couto, U.R.; Vicensoti, A.H.; Georgetti, S.R.; Ida, E.I. Optimisation of soy flour fermentation parameters to produce $\beta$-glucosidase for bioconversion into aglycones. Food Chem. 2014, 152, 56-65.

42. Hwan Nam, D.; Jung Kim, H.; Sun Lim, J.; Heon Kim, K.; Park, C.-S.; Hwan Kim, J.; Lim, J.; Young Kwon, D.; Kim, I.-H.; Kim, J.-S. Simultaneous enhancement of free isoflavone content and antioxidant potential of soybean by fermentation with Aspergillus oryzae. J. Food Sci. 2011, 76, H194-H200.

43. Kim, M.J.; John, K.M.M.; Choi, J.N.; Lee, S.; Kim, A.J.; Kim, Y.M.; Lee, C.H. Changes in secondary metabolites of green tea during fermentation by Aspergillus oryzae and its effect on antioxidant potential. Food Res. Int. 2013, 53, 670-677.

44. Cheng, K.-C.; Wu, J.-Y.; Lin, J.-T.; Liu, W.-H. Enhancements of isoflavone aglycones, total phenolic content, and antioxidant activity of black soybean by solid-state fermentation with Rhizopus spp. Eur. Food Res. Technol. 2013, 236, 1107-1113.

45. Perez-Gregorio, M.R.; Regueiro, J.; Alonso-Gonzalez, E.; Pastrana-Castro, L.M.; Simal-Gandara, J. Influence of alcoholic fermentation process on antioxidant activity and phenolic levels from mulberies (Morus nigra L.). LWT-Food Sci. Technol. 2011, 44, 1793-1801.

46. Hur, S.J.; Lee, S.Y.; Kim, Y.-C.; Choi, I.; Kim, G.-B. Effect of fermentation on the antioxidant activity in plant-based foods. Food Chem. 2014, 160, 346-356.

47. Marazza, J.A.; Garro, M.S.; Savoy de Giori, G. Aglycone production by Lactobacillus rhamnosus CRL981 during soymilk fermentation. Food Microbiol. 2009, 26, 333-339.

48. Georgetti, S.R.; Vicentini, F.T.M.C.; Yokoyama, C.Y.; Borin, M.F.; Spadaro, A.C.C.; Fonseca, M.J.V. Enhanced in vitro and in vivo antioxidant activity and mobilization of free phenolic compounds of soybean flour fermented with different $\beta$-glucosidase-producing fungi. J. Appl. Microbiol. 2009, 106, 459-466.

49. Bhanja, T.; Kumari, A.; Banerjee, R. Enrichment of phenolics and free radical scavenging property of wheat koji prepared with two filamentous fungi. Bioresour. Technol. 2009, 100, 2861-2866. 
50. Liu, C.; Sun, Z.-T.; Du, J.-H.; Wang, J. Response surface optimization of fermentation conditions for producing xylanase by Aspergillus niger SL-05. J. Ind. Microbiol. Biotechnol. 2008, 35, 703-711.

51. Wu, Z.; Song, L.; Huang, D. Food grade fungal stress on germinating peanut seeds induced phytoalexins and enhanced polyphenolic antioxidants. J. Agric. Food Chem. 2011, 59, 5993-6003.

52. Zheng, Z.; Shetty, K. Solid-state bioconversion of phenolics from cranberry pomace and role of Lentinus edodes $\beta$-glucosidase. J. Agric. Food Chem. 2000, 48, 895-900.

53. Hatvani, N.; Mécs, I. Production of laccase and manganese peroxidase by Lentinus edodes on malt-containing by-product of the brewing process. Process Biochem. 2001, 37, 491-496.

54. Panagiotou, G.; Olavarria, R.; Olsson, L. Penicillium brasilianum as an enzyme factory; the essential role of feruloyl esterases for the hydrolysis of the plant cell wall. J. Biotechnol. 2007, 130, 219-228.

55. Żuchowski, J.; Pecio, Ł.; Jaszek, M.; Stochmal, A. Solid-state fermentation of rapeseed meal with the white-rot fungi Trametes versicolor and Pleurotus ostreatus. Appl. Biochem. Biotechnol. 2013, 171, 2075-2081.

56. Tlecuitl-Beristain, S.; Sánchez, C.; Loera, O.; Robson, G.D.; Díaz-Godínez, G. Laccases of Pleurotus ostreatus observed at different phases of its growth in submerged fermentation: Production of a novel laccase isoform. Mycol. Res. 2008, 112, 1080-1084.

57. Correia, R.T.P.; McCue, P.; MagalhÃEs, M.M.A.; MacÊDo, G.R.; Shetty, K. Phenolic antioxidant enrichment of soy flour-supplemented guava waste by Rhizopus oligosporus-mediated solid-state bioprocessing. J. Food Biochem. 2004, 28, 404-418.

58. Vattem, D.A.; Lin, Y.T.; Labbe, R.G.; Shetty, K. Antimicrobial activity against select food-borne pathogens by phenolic antioxidants enriched in cranberry pomace by solid-state bioprocessing using the food grade fungus Rhizopus oligosporus. Process Biochem. 2004, 39, 1939-1946.

59. McCue, P.; Horii, A.; Shetty, K. Solid-state bioconversion of phenolic antioxidants from defatted soybean powders by Rhizopus oligosporus: Role of carbohydrate-cleaving enzymes. J. Food Biochem. 2003, 27, 501-514.

60. Ajila, C.M.; Brar, S.K.; Verma, M.; Tyagi, R.D.; Valéro, J.R. Solid-state fermentation of apple pomace using Phanerocheate chrysosporium - Liberation and extraction of phenolic antioxidants. Food Chem. 2011, 126, 1071-1080.

61. Coghe, S.; Benoot, K.; Delvaux, F.; Vanderhaegen, B.; Delvaux, F.R. Ferulic acid release and 4-vinylguaiacol formation during brewing and fermentation: Indications for feruloyl esterase activity in Saccharomyces cerevisiae. J. Agric. Food Chem. 2004, 52, 602-608.

62. Restuccia, C.; Muccilli, S.; Palmeri, R.; Randazzo, C.L.; Caggia, C.; Spagna, G. An alkaline $\beta$-glucosidase isolated from an olive brine strain of Wickerhamomyces anomalus. FEMS Yeast Res. 2011, 11, 487-493.

63. Rao, K.V.; Weisner, N.T. Microbial transformation of suercetin by Bacillus cereus. Appl. Environ. Microbiol. 1981, 42, 450-452.

64. Gao, C.; Mayon, P.; MacManus, D.A.; Vulfson, E.N. Novel enzymatic approach to the synthesis of flavonoid glycosides and their esters. Biotechnol. Bioeng. 2000, 71, 235-243. 
65. Bertrand, A.; Morel, S.; Lefoulon, F.; Rolland, Y.; Monsan, P.; Remaud-Simeon, M. Leuconostoc mesenteroides glucansucrase synthesis of flavonoid glucosides by acceptor reactions in aqueous-organic solvents. Carbohydr. Res. 2006, 341, 855-863.

66. Ibrahim, A.; Khalifa, S.I.; Khafagi, I.; Youssef, D.T.; Khan, S.; Mesbah, M.; Khan, I. Microbial metabolism of biologically active secondary metabolites from Nerium oleander L. Chem. Pharm. Bull. 2008, 56, 1253-1258.

67. Miyakoshi, S.; Azami, S.; Kuzuyama, T. Microbial glucosylation of flavonols by Cunninghamella echinulata. J. Biosci. Bioeng. 2010, 110, 320-321.

68. Zi, J.; Valiente, J.; Zeng, J.; Zhan, J. Metabolism of quercetin by Cunninghamella elegans ATCC 9245. J. Biosci. Bioeng. 2011, 112, 360-362.

69. Chen, S.; Xing, X.-H.; Huang, J.-J.; Xu, M.-S. Enzyme-assisted extraction of flavonoids from Ginkgo biloba leaves: Improvement effect of flavonol transglycosylation catalyzed by Penicillium decumbens cellulase. Enzym. Microb. Technol. 2011, 48, 100-105.

70. Di Gioia, D.; Strahsburger, E.; Lopez de Lacey, A.M.; Bregola, V.; Marotti, I.; Aloisio, I.; Biavati, B.; Dinelli, G. Flavonoid bioconversion in Bifidobacterium pseudocatenulatum B7003: A potential probiotic strain for functional food development. J. Funct. Food 2014, 7, 671-679.

71. Kaur, A.; Singh, S.; Singh, R.S.; Schwarz, W.H.; Puri, M. Hydrolysis of citrus peel naringin by recombinant $\alpha$-L-rhamnosidase from Clostridium stercorarium. J. Chem. Technol. Biotechnol. 2010, 85, 1419-1422.

72. Landete, J.M.; Curiel, J.A.; Rodríguez, H.; de las Rivas, B.; Muñoz, R. Aryl glycosidases from Lactobacillus plantarum increase antioxidant activity of phenolic compounds. J. Funct. Foods 2014, 7, 322-329.

73. You, H.J.; Ahn, H.J.; Ji, G.E. Transformation of rutin to antiproliferative quercetin-3-glucoside by Aspergillus niger. J. Agric. Food Chem. 2010, 58, 10886-10892.

74. Liu, J.-Y.; Yu, H.-S.; Feng, B.; Kang, L.-P.; Pang, X.; Xiong, C.-Q.; Zhao, Y.; Li, C.-M.; Zhang, Y.; Ma, B.-P. Selective hydrolysis of flavonoid glycosides by Curvularia lunata. Chin. J. Nat. Med. 2013, 11, 684-689.

75. Hund, H.-K.; Breuer, J.; Lingens, F.; Hüttermann, J.; Kappl, R.; Fetzner, S. Flavonol 2,4-dioxygenase from Aspergillus niger DSM 821, a type 2 CuII-containing glycoprotein. Eur. J. Biochem. 1999, 263, 871-878.

76. Udupa, S.R.; Banerji, A.; Chadha, M.S. Microbiological transformations of flavonoids-II: Transformations of $( \pm$ ) flavanone. Tetrahedron 1969, 25, 5415-5419.

77. De, M. B. Costa, E.M.; Pimenta, F.C.; Luz, W.C.; de Oliveira, V. Selection of filamentous fungi of the Beauveria genus able to metabolize quercetin like mammalian cells. Braz. J. Microbiol. 2008, 39, 405-408.

78. Araújo, K.C.F.; de M. B. Costa, E.M.; Pazini, F.; Valadares, M.C.; de Oliveira, V. Bioconversion of quercetin and rutin and the cytotoxicity activities of the transformed products. Food Chem. Toxicol. 2013, 51, 93-96.

79. Edyta, K.-S.; Tomasz, J. Microbial transformations of 7-hydroxyflavanone. Sci. World J. 2012 , 254929:1-254929:8.

80. Herath, W.; Khan, I.A. Microbial metabolism. Part 13: Metabolites of hesperetin. Bioorg. Med. Chem. Lett. 2011, 21, 5784-5786. 
81. Ma, B.; Zeng, J.; Shao, L.; Zhan, J. Efficient bioconversion of quercetin into a novel glycoside by Streptomyces rimosus subsp. rimosus ATCC 10970. J. Biosci. Bioeng. 2013, 115, 24-26.

82. Slana, M.; Žigon, D.; Makovec, T.; Lenasi, H. The response of filamentous fungus Rhizopus nigricans to flavonoids. J. Basic Microbiol. 2011, 51, 433-441.

83. Park, J.S.; Rho, H.S.; Kim, D.H.; Chang, I.S. Enzymatic preparation of kaempferol from green tea seed and its antioxidant activity. J. Agric. Food Chem. 2006, 54, 2951-2956.

84. Turner, C.; Turner, P.; Jacobson, G.; Almgren, K.; Waldeback, M.; Sjoberg, P.; Karlsson, E.N.; Markides, K.E. Subcritical water extraction and [small beta]-glucosidase-catalyzed hydrolysis of quercetin glycosides in onion waste. Green Chem. 2006, 8, 949-959.

85. Yadav, V.; Yadav, P.K.; Yadav, S.; Yadav, K.D.S. $\alpha$-L-Rhamnosidase: A review. Process Biochem. 2010, 45, 1226-1235.

86. Avila, M.; Jaquet, M.; Moine, D.; Requena, T.; Pelaez, C.; Arigoni, F.; Jankovic, I. Physiological and biochemical characterization of the two $\alpha$-L-rhamnosidases of Lactobacillus plantarum NCC245. Microbiology 2009, 155, 2739-2749.

(C) 2014 by the authors; licensee MDPI, Basel, Switzerland. This article is an open access article distributed under the terms and conditions of the Creative Commons Attribution license (http://creativecommons.org/licenses/by/4.0/). 